\title{
EFFICIENT FACE RECOGNITION USING CONVOLUTIONAL NEURAL NETWORKS
}

\author{
T.Syed Akheel ${ }^{1}$, Dr.S.A.K Jilani ${ }^{2}$
}

Abstract-In this paper we present a new technique for face recognition with Convolutional auto-encoder (CAE) with neural network. The face recognition is the crucial step for many applications like surveillance, access control or human-computer interaction. Existing Convolution Neural Network (CNN) based methods for face recognition are not robust and reliable. We propose a novel face recognition approach based Convolution neural network and Siamese network. As a standout amongst the best uses of picture examination and comprehension, face acknowledgment has as of late gotten huge consideration, particularly amid the past quite a long while. No less than two reasons represent this pattern: the first is the extensive variety of business and law authorization applications, and the second is the accessibility of achievable advancements since years of exploration. Despite the fact that present machine acknowledgment frameworks have achieved a specific level of development, their prosperity is constrained by the conditions forced by numerous genuine applications. For instance, acknowledgment of face pictures procured in an open air environment with changes in light or posture remains a great extent unsolved issue. At the end of the day, current frameworks are still far away from the ability of the human discernment framework. In this paper we present a survey of latest state of the art techniques for face recognition

Key words: Convolution neural networks, Siamese network, Convolutional auto-encoder

\section{I.INTRODUCTION}

There are numerous possible applications for facial image processing algorithms. The possible application for face authentication are mainly concerned with access control, e.g. restricting the physical access to a building, such as a corporate building, a secured zone of an airport etc. This principle could equally be applied to the access to systems, automatic teller machines, mobile phones, Internet sites etc. Video surveillance is another application for face identification facial recognition framework is a PC application fit for recognizing or confirming a man from a picture or a video frame from a video source. One of the approaches to do this is by selecting facial features from the picture and a facial database. It is ordinarily utilized as a part of security frameworks and can be contrasted with different biometrics, for example, unique finger impression recognition or eye iris recognition frameworks. Some facial recognition systems distinguish facial feature vectors by extracting points of interest, or elements, from a picture of the subject's face. For instance, a calculation may dissect the relative position, size, and/or state of the eyes, nose, cheekbones, and jaw. These elements are then used to look for different pictures with coordinating features. Other systems normalize a collection of face pictures and after that pack the face information, just sparing the information in the picture that is helpful for face recognition. A test picture is then compared with the face data. One of the most punctual fruitful systems depends on layout coordinating techniques connected to an arrangement of remarkable facial components, giving a kind of packed face representation. Recognition techniques can be isolated into two fundamental methodologies, geometric, which takes distinguishing features into account for recognizing, or photometric, which is a measurable methodology that distills a picture into qualities and contrasts the qualities and formats to dispense with differences. Mainstream acknowledgment calculations incorporate Principal Component Analysis utilizing eigenfaces, Linear Discriminate Analysis, Elastic Bunch Graph Matching utilizing the Fisherface calculation, the Hidden Markov demonstrate, the Multilinear Subspace Learning utilizing tensor representation,

\footnotetext{
${ }^{1}$ Dept. of Electronics \& Communication Engg., Rayalaseema University Kurnool, Andhra Pradesh

${ }^{2}$ Dept. Of Electronics \& Communication Engg, , Madanapalle Institute of Technology \& science, Madanapalle, Andhra Pradesh
} 
and the neuronal spurred element connection coordinating. A lot of research is conducted in this domain. The techniques are broadly divided into categories:

i) Image based: The facial images are directly used for the recognition.

ii) Feature based: facial features are extracted and then sent to the recognition engine.

In this study we combine both the classifications for face recognition. Under image based techniques we use neural network techniques for image based facial recognition. The feature extraction methods for feature vectors form the facial analysis and use those for recognition.

\section{II.REALTED WORK}

It is defined as a computing system made up of a number of simple, highly interconnected processing elements, which process information by their dynamic state response to external inputs. Artificial Neural Networks (ANNs) $[22,23,24]$ are processing devices that are modeled after the neural structure of the mammalian cerebral cortex on smaller scales. Neural networks are typically organized in layers. Layers are made up of a number of interconnected 'nodes' which contain an 'activation function'. Patterns are given to the network through the 'input layer' which communicates to one or more 'hidden layers' where the actual processing is done through a system of 'weighted connections'. The hidden layers then link to an 'output layer' where the answer is given to the output. L. B. Almeida,. Et.al [1] presented the stochstic optimization for features extractiona using neural networks

S.-I. Amari, Et.al [2] has proposed a new learning algorithm for blind source separation method helps learning the huge database In D. S. Touretzky Et.al [3] which deals with Advances in Neural Information Processing Systems further. F. R. Bach and M. I. Jordan Et.al [10] uses the Kernel independent component analysis and similarly video based biometric person authentication are suggested in $[11,12,14,15]$, The best learning algorithms using convolutional networks are presented in Lecun Et.al.

Feed Forward Neural Network:A Feed Forward Neural network is an artificial neural network where the connections do not form a cycle. This network was the first and simplest form of the artificial neural network devised. In this network the information moves only in one direction, forward from the input nodes through the hidden nodes and then to the output nodes.

III.PROPOSED METHOD FACE RECOGNTION:

INPUT: Given a training set $X=\left\{X^{i} \mathrm{~m}\right\}$ consisting of $\mathrm{N}$ face images from ' $\mathrm{C}$ ' classes, with $\mathrm{N}_{\mathrm{i}}$ face images per class specified with their class label, and $\mathrm{X}=\left\{\mathrm{Xi}^{\mathrm{m}}\right\}$ denoting the $\mathrm{m}^{\text {th }}$ sample from the $\mathrm{i}^{\text {th }}$ class.

OUTPUT: A classification function that assign the testing sample ' $\mathrm{x}$ ' correctly to a class label $\mathrm{r} \varepsilon \mathrm{C}$, where $\mathrm{C}=$ $\{1, \ldots . c\}$ denotes a label set with $\mathrm{c}$ classes.

Step 1: $\quad$ Face Normalization:

SUMMARY OF THE ALGORITHM:

1. Initialize the data vectors.

2. Set Hyper Parameters

a. number of input units

b. number of hidden

c. number of softmax output units

3. Initialization of weights

4. Make batches from the input data

5. Loop over epochs

6. Loop over batches

7. Step 1: Forward Propagation: Compute state of each layer in the networks given the input batch and all weights and biases.

a. Subtract maxima: Adding or subtracting the same constant from each input to a softmax unit does not affect the outputs. Here we are subtracting maximum to make all inputs $<=0$. This prevents overflows when computing their exponents.

b. $\quad$ Normalize to get probability distribution

c. Measure Cross Entropy Loss

d. current batch entropy loss

\section{Step 2: Back Propagation Is Employed}

Back Propagation: Back propagation is employed to compute derivative of loss function w.r.t weights in neural network.

e. First compute deltas partial derivative of cross entropy loss function w.r.t inputs to softmax units partial derivative of cross entropy loss function w.r.t inputs to hidden units. 
f. Then compute gradients partial derivative of cross entropy loss function w.r.t hid2out_weights

STEP 3: TESTING

In the testing part for each face image gets the near frontal face using the SEGNET trained parameters.

\section{IV.COMPARISON AND RESULTS}

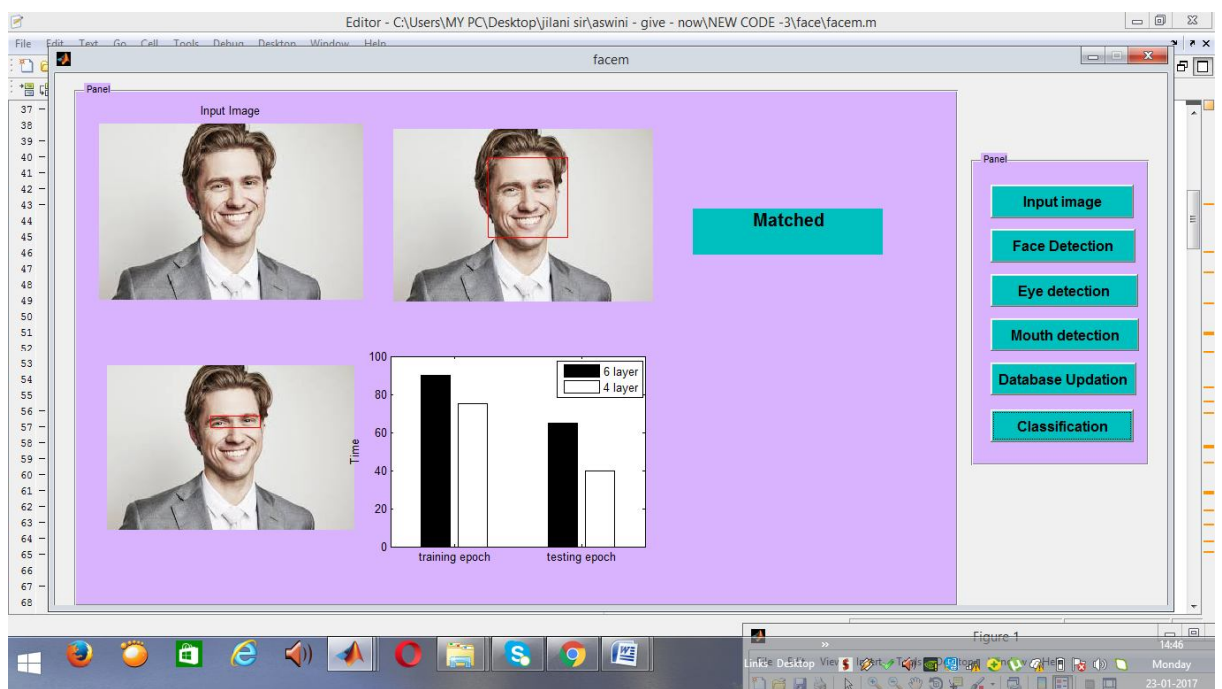

Figure 1. Data Updating Process Using Gui

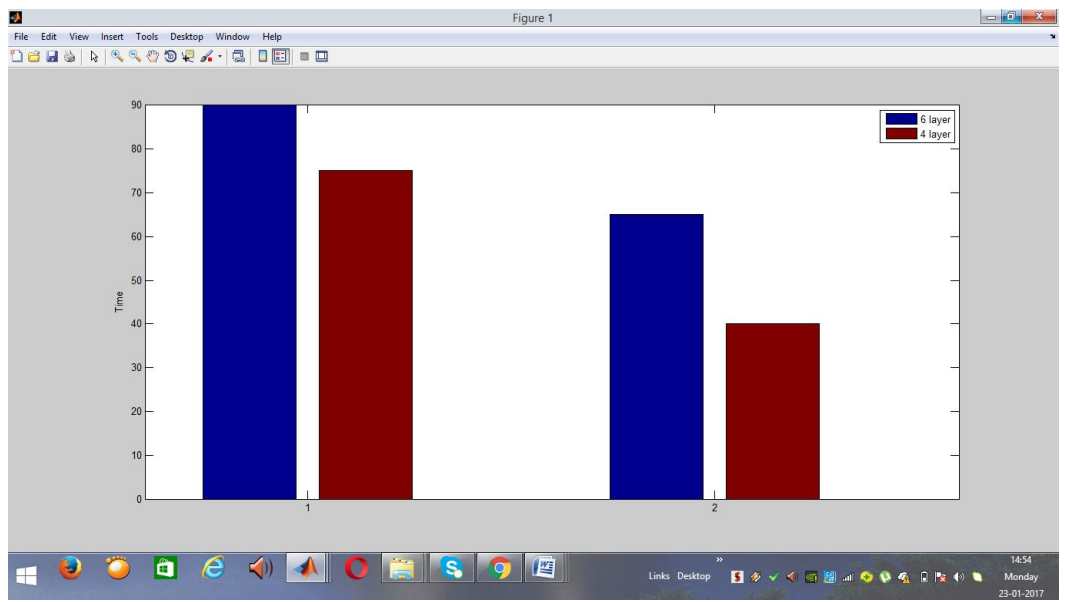

Figure 2. Convolution layer epochs

Before the data is processed it should be normalized, and for updating a normal GUI is interfaced as shown on fig 1, when the convolution is performed the epochs are calculated as shown in fig 2 .The mean values and standard deviation can also be calculated as shown in the table 2 . 


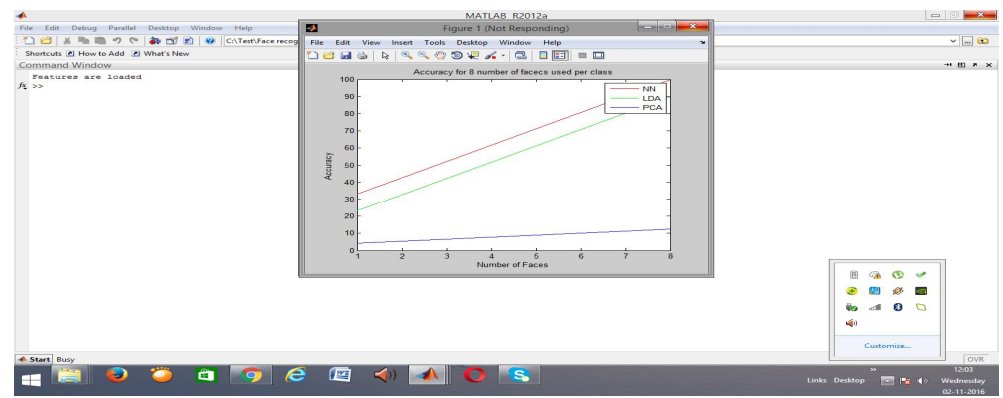

Figure 3.Showing The Accuracy For 8 No Of Faces Used Per Class

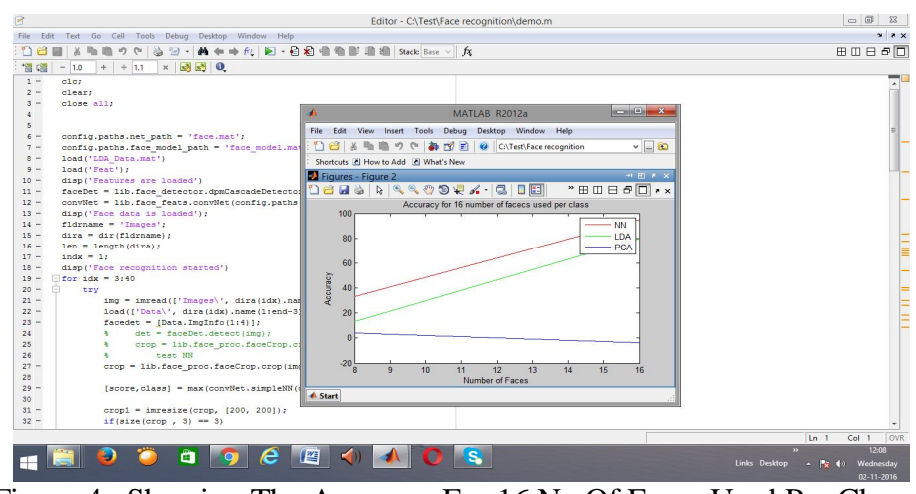

Figure 4 . Showing The Accuracy For 16 No Of Faces Used Per Class

\section{V.COMPARSION OF ACCURACIES /ELAPSED TIME FOR VARIOUS ALGORITHMS}

\begin{tabular}{|l|l|l|l|}
\hline S. No & Methods & Database & Time in seconds \\
\hline 1 & LRC METHODS & Orl/Yale & 10.07 \\
\hline 2 & LCDRC METHOD & Orl/Yale & 10.07 \\
\hline 3 & $\begin{array}{l}\text { PROPOSED } \\
\text { MONVOLUTION } \\
\text { METHOD }\end{array}$ & Orl/Yale & 2.820 \\
\hline
\end{tabular}

Table: 1 Accuracy Comparison Data

Table 1 gives the comparison table with other systems with regression methods [25,26].

VI. CALCULATION OF MEAN AND STANDARD DEVCIATION FOR THE YOUTUBE IMAGES:

\begin{tabular}{|l|l|l|}
\hline S.NO & MEAN & STANDARD DEVIATION \\
\hline 1. & $\mathrm{M}=126.0813$ & $\mathrm{~S}=53.217$ \\
\hline
\end{tabular}

Table: 2 Accuracy Comparison Data

Table 2 gives the present system mean and standard deviation calculation for the present dataset.

Here we present the analysis on two types of database:

1. Labelled faces in the wild.

2. YouTube face database.

For the calculation of loss of the system we use two types of loss functions. Soft max regression based method uses the L2 distance comparison. The distance between the actual face and the near frontal face is derived using the metric L2 distance. Another type of method used for the loss is Embedding loss as the loss function. The following are the accuracies given by two different types of databases in comparison with the present technique and having two different types of loss functions. . It is observed that the CNN is giving good accuracy results but not as good as the deep learning networks. The Deep learning networks are giving good results when compared to $\mathrm{CNN}$ based face recognition. 
Table 3: Accuracies Compared To CAE with Siamese Network and CNN, VGG Deep Learning Network For Different Types of Datasets

\begin{tabular}{|l|l|l|l|}
\hline Training method & Loss function & $\begin{array}{l}\text { Labeled faces in the } \\
\text { wild }\end{array}$ & YouTube faces dataset \\
\hline CNN & $\begin{array}{l}\text { Softmax (L2 distance } \\
\text { comparision) }\end{array}$ & 95.4 & 91.7 \\
\hline VGG & $\begin{array}{l}\text { Softmax (L2 distance } \\
\text { comparision) }\end{array}$ & 97.27 & 92.8 \\
\hline CAE with Siamese network & $\begin{array}{l}\text { Softmax (L2 distance } \\
\text { comparision) }\end{array}$ & 99.3 & 94.6 \\
\hline CNN & Embedding Loss & 96.5 & 94.3 \\
\hline CGG & Embedding Loss & 99.13 & 97.4 \\
\hline CAE with Siamese network & Embedding Loss & 99.45 & 98.7 \\
\hline
\end{tabular}

The Above shows the accuracies compared to CAE with Siamese network and CNN, VGG deep learning network for different types of datasets. The CNN based face recognition will create a face image which is near to the mean image of a particular person according to the distance metric and then the training of the algorithm is done which is same as the Present CAE with Siamese network. But the only difference is that the training of the algorithm is taken care by the $\mathrm{CNN}$ with auto-encoder will create the near frontal face for each individual. The CAE with Siamese network has the highest accuracy for two different types of loss functions and using the two datasets when compared to the other networks.

Table 3 gives the benchmarking results of our work on Different types of database. It shows that our design has outperformed some reported results..

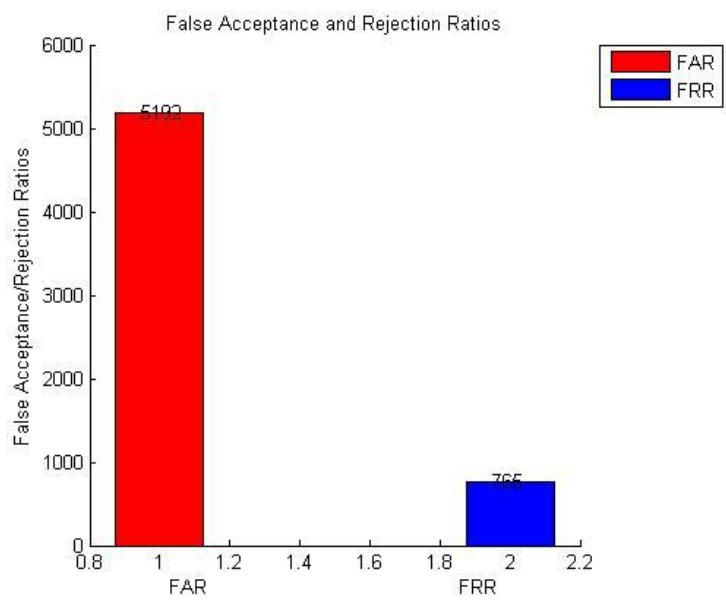

Figure 5: FAR and FRR using CAE

A system's FAR typically is stated as the ratio of the number of false acceptances divided by the number of identification attempts and a system's FRR typically is stated as the ratio of the number of false recognitions divided by the number of identification attempts. Figure 7 Here, we discusses biometric recognition systems, describes False Acceptance rejection ratio and explains how the quality of two systems can be compared objectively. 


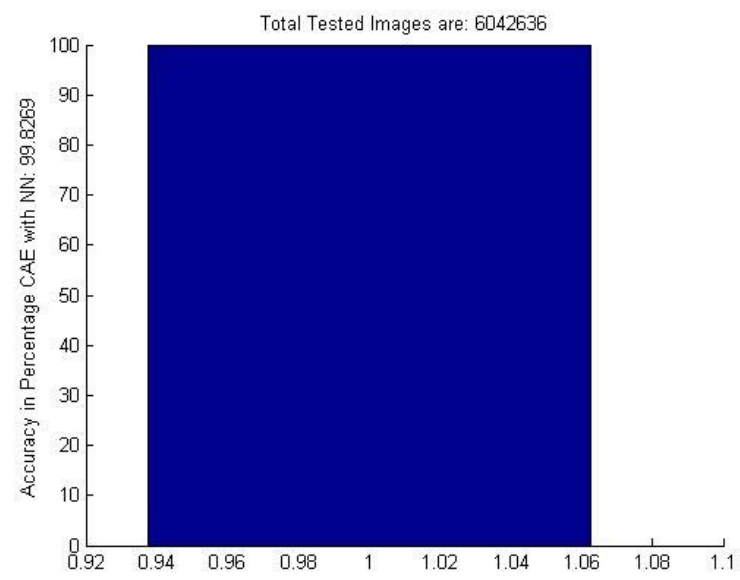

Figure 6: Accuracy Rate using CAE

Figure 6 shows discussed Accuracy percentage with CAE and total test images based on face recognition.

\section{VI.CONCLUSION}

Experimental results proved that the Convolutional auto encoder can perform far better than the LDA, PCA, and neural networks, when it comes to handle large and most challenging data base, Convolution Neural networks has performs remarkable results.

\section{REFERENCES}

[1] L. B. Almeida, T. Langlois, J. D. Amaral, and A. Plakhov. Parameter adaptation in stochastic optimization. In D. Saad, editor, On-Line Learn-ing in Neural Networks, chapter 6. Cambridge University Press, 1999.

[2] S.-I. Amari, A. Cichocki, and H. Yang. A new learning algorithm for blind source separation. In D. S. Touretzky, M. C. Moser, and M. E. Hasselmo ,editors, Advances in Neural Information Processing Systems, volume 8,pages 757-763. MIT Press, Cambridge, MA, 1996.

[3] G. Arulampalam and A. Bouzerdoum. A generalized feed forward neural network architecture for classification and regression. Neural Networks,16:561-568, 2003.

[4] A. Azarbayejani, T. Starner, B. Horowitz, and A. Pentland. Visually controlled graphics. IEEE Transactions on Pattern Analysis and Machine Intelligence, 15(6):602-605, June 1993.

[5] S. O. Ba and J. M. Odobez. A probabilistic framework for joint head tracking and pose estimation. In Proceedings of the 17th International Confer-ence on Pattern Recognition, volume 4, pages 264-267, August 2004.

[6] F. R. Bach and M. I. Jordan. Kernel independent component analysis .Journal of Machine Learning Research, 3:1-48, 2002.

[7] E. Bailly-Bailli`ere, S. Bengio, F. Bimbot, M. Hamouz, J. Kittler, J. Mari'ethoz,J. Matas, K. Messer, V. Popovici, F. Por'ee, B. Ruiz, and J.-P. Thiran. The BANCA database and evaluation protocol. In proceedings of the Fourth International Conference on Audio- and Video-Based Biometric Person Authentication, pages 625-638, 2003.

[8] S. Baker and I. Matthews. Equivalence and efficiency of image alignment algorithms. In Computer Vision and Pattern Recognition, volume 1, pages1090-1097, 2001.

[9] P. Baldi and K. Hornik. Neural networks and principal component analysis :Learning from examples without local minima. IEEE Transactions on Neural Networks, 2:53-58, 1988.

[10] S. Baluja. Probabilistic modeling for face orientation discrimination: Learning from labeled and unlabeled data. In Neural Information Processing Systems, 1998

[11] S. Baluja and H. A. Rowley. Boosting sex identification performance .International Journal of Computer Vision, 71(1):111-119, 2007.

[12] M. S. Bartlett, J. R. Movellan, and T. J. Sejnowski. Face recognition byin dependent component analysis. IEEE Transactions on Neural Networks,13(6):1450-1464, 2002. 
[13] R. Basri and D. Jacobs. Lambertian reflectance and linear subspaces. In International Conference on Computer Vision, volume 2, pages 383-390,2001.R. Battiti. Accelerated back propagation learning: Two optimization methods. Complex Systems, 3:331-342, 1989.

[14] P. Belhumeur, J. Hespanha, and D. Kriegman. What is the set of images of an object under all possible lighting conditions. International Journal of Computer Vision, 28(3):245-260, 1998.

[15] P. Belhumeur, J. Hespanha, and D. Kriegman. What is the set of images of an object under all possible lighting conditions. International Journal of Computer Vision, 28(3):245-260, 1998.

[16] P. N. Belhumeur, J. P. Hespanha, and D. J. Kriegmann. Eigen faces vs Fisher faces: Recognition using class specific linear projection. IEEE Transactions on Pattern Analysis and Machine Intelligence, 17(7):711-720, 1997.

[17] A. Bell and T. Sejnowski. An information maximization approach to blind separation and blind deconvolution. Neural Computation, 7:1129-1159,1995.

[18] C. Ben AbdelKader and P. Griffin. A local region-based approach to gender classification from face images. In Proceedings of the IEEE Conference on Computer Vision and Pattern Recognition, 2005.

[19] Y. Bengio, Y. LeCun, and D. Henderson. Globally trained hand written word recognizer using spatial representation, space displacement neural networks and hidden markov models. In Advances in Neural Information Processing Systems, volume 6, pages 937-944. Morgan Kaufmann, 1994.

[20] T. L. Berg, A. C. Berg, J. Edwards, M. Maire, R. White, Yee-Whye Teh,E. Learned-Miller, and D. A. Forsyth. Names and faces in the news. In Proceedings of the International Conference on Computer Vision and Pattern Recognition, volume 2, pages 848-854, 2004.

[21] A Convolution Neural Networks by Kalil hani and liew shang suang convolution neural network approach for face recogntion

[22] Dimensionality Reduction by Learning an Invariant Mapping Raia Hadsell, Sumit Chopra, Yann LeCunThe Courant Institute of Mathematical Sciences New York University, 719 Broadway, New York, NY 1,USA

[23] Learning Algorithms For Classification A Comparison N Hand Writteen Digital Recogntion, yabb lecun ,L.D jackel,leon bottou,corinna cortes

[24] Signature Verification using a "Siamese" Time Delay Neural Network Jane Bromley, Isabelle Guyon, Yann LeCun, Eduard Sickinger and Roopak Shah AT\&T Bell Laboratories

[25] SegNet: A Deep Convolutional Encoder-Decoder Architecture for Image Segmentation Vijay Badrinarayanan, Alex Kendall, Roberto Cipolla, Senior Member, IEEE,

[26] Xiaochao Qu, Suah Kim, Run Cui, Hyoung Joong Kim, "Linear collaborative discriminant regression classification for face recognition", Journal of Visual Communication and Image Representation, Vol. 31, p. 312-319, 2015.

[27] S.-M. Huang and J.-F. Yang, "Linear discriminant regression classification for face recognition,"Signal Processing Letters, IEEE, vol. 20, no. 1, pp. 91-94, 2013.

[28] Learning Algorithms For Classification A Comparison N Hand Writteen Digital Recogntion, Lecun Et.al. 